\title{
Classification of mesh fiber exposure: reply
}

\author{
Bernard T. Haylen
}

Published online: 5 May 2011

(C) The International Urogynecological Association 2011

\section{Dear Editor,}

On behalf of the authors, I thank Dr Al-Badr for his interest in the document [1]: "An International Urogynecological Association (IUGA) / International Continence Society (ICS) joint Terminology and Classification of complications related directly to the insertion of prostheses (meshes, implants, tapes) and grafts in female pelvic floor surgery". It is pleasing the report has been found to be useful.

The main point made by Dr Al-Badr [2] appears to concern the inclusion of "mesh fibre palpation" in category 1 of the classification [1] rather than category 2 . The issue rests on the definition of (vaginal) "separation", which is currently applicable, to "exposures" in categories 2 and 3 of the classification but not to category 1 complications. Dr Al-Badr appears to argue that a "separation" of vaginal epithelium by one fibre is still a "separation". The definition of "separation" used in the Terminology section of the Report [1] is that of "physically disconnected". It may be hard to assert that vaginal epithelium pierced by one or more than one fibre of a prosthesis is "physically disconnected". The Report maintains that there needs to be clear physical disconnection ("separation") of vaginal epithelium with visualization of vaginal mesh to create an exposure (categories 2 and 3).

Dr Al-Badr makes a further point [2] that the classification [1] does not distinguish between mesh fibre palpation under the vaginal epithelium and mesh palpation and/or visualization through the epithelium. The suggested argument is that the management may well be different, which may perhaps, at times, be the case. It is agreed that mesh fibres felt under the epithelium without being seen (or felt) in the vagina qualifies for category $1 \mathrm{~A}$ (especially as it is likely to be asymptomatic and generally requiring no treatment). For the reasons outlined above in relation to the definition of "separation", mesh fibres seen or felt through the vaginal epithelium would still only qualify as category 1 . The category would be $1 \mathrm{~A}$ if there were no symptoms or $1 \mathrm{~B}$ is there were symptoms (possibly requiring treatment), with a common symptom being dyspareunia to the partner. The classification does then distinguish between the scenarios presented by Dr Al-Badr on the basis of the likely presence or absence of symptoms and the possible associated need for treatment.

Conflicts of interest None.

\section{References}

1. Haylen BT, Freeman RM, Swift SE, Cosson M, Davila GW, Deprest J, Dwyer PL, Fatton B, Kocjancic E, Lee J, Maher C, Petri E, Rizk DE, Sand PK, Schaer GN, Webb RJ (2011) An International Urogynecological Association (IUGA) / International Continence Society (ICS) joint Terminology and Classification of the complications related directly to the insertion of prostheses (meshes, implants, tapes) and grafts in female pelvic floor surgery. Int Urogynecol J 22(1):3-15. doi:10.1007/s00192-010-1324-9

2. Al-Badr A (2011) Classification of mesh fibre exposure: comment. Int Urogynecol J. doi:10.1007/s00192-011-1436-x
B. T. Haylen $(\bowtie)$

St. Vincent's Clinic, 904/438 Victoria Street, Darlinghurst NSW 2010, Australia

e-mail: haylen@optusnet.com.au 